\title{
Coordination of kidney organogenesis by Wnt signaling
}

\author{
Kimmo Halt • Seppo Vainio
}

Received: 6 June 2013 / Revised: 25 October 2013 / Accepted: 12 December 2013 / Published online: 21 January 2014

(C) The Author(s) 2014. This article is published with open access at Springerlink.com

\begin{abstract}
Several Wnt proteins are expressed in the embryonic kidney during various stages of development. Gene knockout models and ex vivo studies have provided strong evidence that Wnt-mediated signals are essential in renal ontogeny. Perhaps the most critical factors, Wnt9b and Wnt4, function during the early phase when the cap mesenchyme is induced to undergo morphogenesis into a nephron. Wnt11 controls early ureteric bud branching and contributes to the final kidney size. In addition to its inductive role, later on Wnt9b plays a significant role in the convergent extension of the tubular epithelial cells, while Wnt4 signaling controls smooth muscle cell fates in the medulla. Wnt7b has a specific function together with its likely antagonist Dkk1 in controlling the morphogenesis of the renal medulla. The signaltransduction mechanisms of the Wnts in kidney ontogeny have not been resolved, but studies characterizing the downstream signaling pathways are emerging. Aberrant Wnt signaling may lead to kidney diseases ranging from fatal kidney agenesis to more benign phenotypes. Wnt-mediated signaling regulates several critical aspects of kidney development from the early inductive stages to later steps of tubular epithelial maturation.
\end{abstract}

Keywords Wnt signaling $\cdot$ Tubule induction $\cdot$ Nephron segmentation $\cdot$ PCP

$\mathrm{K}$. Halt $\cdot \mathrm{S}$. Vainio

The Centre of Excellence in Cell-Extracellular Matrix Research, Oulu, Finland

K. Halt $\cdot$ S. Vainio

Biocenter Oulu, Oulu, Finland

K. Halt $\cdot$ S. Vainio $(\bowtie)$

Laboratory of Developmental Biology, Department of Medical Biochemistry and Molecular Biology, Institute of Biomedicine, University of Oulu, PO Box 5000, 90014 Oulu, Finland

e-mail: seppo.vainio@oulu.fi

\section{Introduction}

The definitive kidney of mammals, the metanephros, appears as a morphologically distinguishable rudiment around midgestation [1]. The early metanephros is composed of the epithelial ureteric bud (UB), an outgrowth from the adjacent Wolffian duct, and the metanephric mesenchyme (MM). The $\mathrm{MM}$ is divided into the cap mesenchyme (CM), which is adjacent to the UB, and the cortical interstitial stroma, which surrounds the CM (Fig. 1). Fate-mapping studies have demonstrated that the metanephric cells originate from the intermediate mesoderm, which also gives rise to the pronephros and mesonephros. Both largely regress in mammals but construct a functional kidney in lower vertebrates $[1,2]$.

After the establishment of the metanephros, the kidney organogenesis proceeds with iterative branching of the UB and subsequent differentiation of the nephrons from the $\mathrm{CM}$ adjacent to the tips of the UB. The differentiation of the nephrons from the progenitor cells begins as interplay between the CM and the UB leading to induction of the CM and subsequent transition of the induced cells into a pretubular aggregate, which undergoes a mesenchyme-toepithelium transformation (MET) to establish a renal vesicle, which in turn becomes connected to the collecting duct system generated by the UB $[3,4]$.

The renal vesicle matures into a functional nephron through comma- and S-shaped body stages (Fig. 2). The primordial nephron becomes elongated along the corticomedullary axis and forms morphologically distinguishable segments containing a Bowman's capsule, a proximal convoluted tube, a loop of Henle, a distal convoluted tube, and a connecting tube. At the vascular pole, the nephron connects to the glomerular tuft composed of endothelial and mesangial cells, whereas the distal part joins with the collecting duct.

Generation and analysis of several gene-targeted mouse models has identified elements of a transcriptional program 


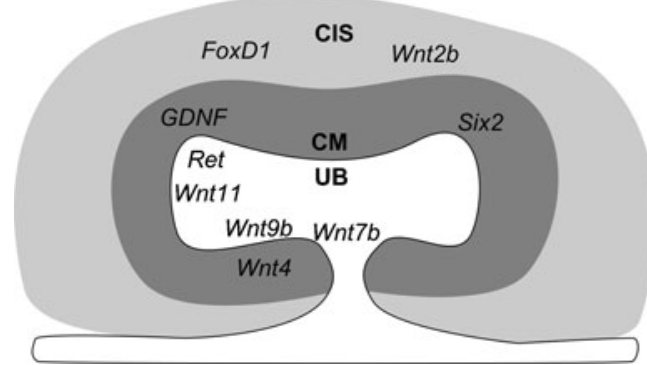

Fig. 1 Schematic micrograph of the embryonic kidney. A ureteric bud (UB, white) invades the metanephric mesenchyme, which consists of the cap mesenchyme (CM, dark grey) and the cortical interstitial mesenchyme (CIS, light grey). Six 2 and FoxD1 transcription factors mark the $\mathrm{CM}$ and CIS, respectively. Wnt $9 b$ is expressed in the UB where its paracrine action induces Wnt 4 expression in the ventral CM. Wnt 11 in the UB tips establishes a positive signaling loop with the GDNF/Ret system to promote UB branching. $W n t 7 b$ is expressed in the UB where it regulates the development of the renal medulla. The role of $W n t 2 b$ in the CIS is unknown

behind nephrogenesis. The nephron stem cell pool marked by sine oculis-related homeobox 2 (Six2) maintains the putative self-renewal potential of these cells and prevents their premature epithelization $[5,6]$, but how is still poorly understood. Expression of Six 2 mRNA is rapidly down-regulated in the pre-tubular cell aggregates [7], while the respective protein persists in the proximal pole of the renal vesicle [8]. Cited1 expression is down-regulated during the $\mathrm{CM}$ induction and thus Cited 1 specifically marks the dorsal un-induced CM that is still in the stem cell stage [7] (Fig. 3).

The Wnt signals play an important role in several distinct processes during kidney development. Evidence suggests that they are involved during initial induction of the $\mathrm{CM}$ and during the maturation of the nephron. Deficiencies in Wnt signaling may result in manifestations ranging from serious renal defects to more benign phenotypes.

The most thorough insight into vertebrate kidney development was obtained by using transgenic mice. These in vivo molecular genetic studies are in line with studies in the Xenopus laevis and of human diseases, collectively suggesting a conserved role for Wnt signaling in vertebrate kidney
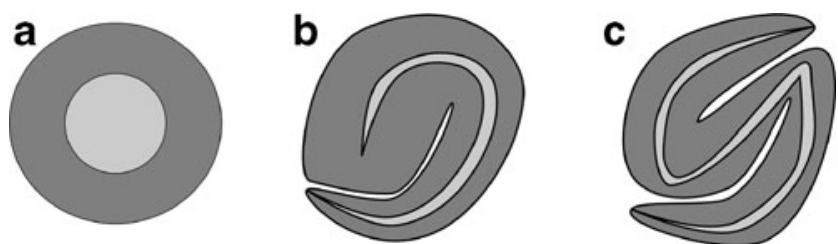

Fig. 2 Nephron morphogenesis. As a consequence of mesenchyme-toepithelium transition, a lumen containing renal vesicle (a) is assembled from the pre-tubular aggregate. A cleft forms within the epithelium of the renal vesicle turning it into a comma-shaped body (b). After another cleft formation step in the distal comma-shaped body an S-shaped body stage emerges (c). The distal part of the S-shaped body connects with the ureteric bud (UB)-derived collecting duct system while the proximal part forms the Bowman's capsule

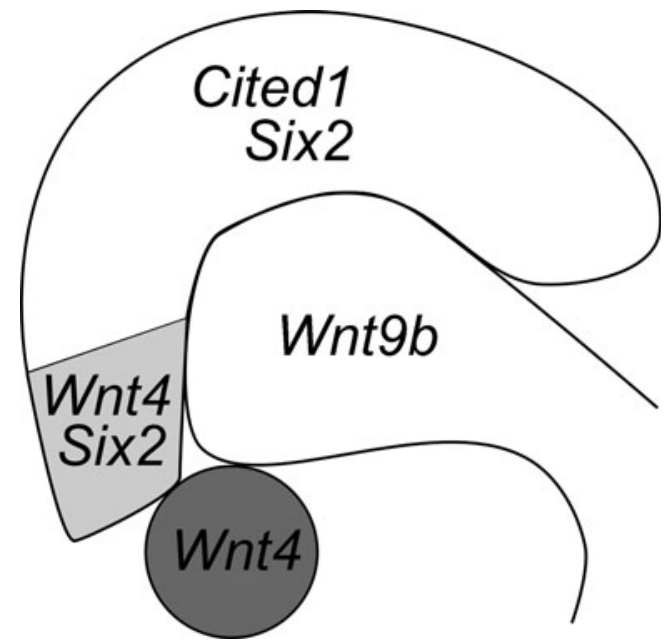

Fig. 3 The relation between the ureteric bud (UB) tip and the cap mesenchyme $(\mathrm{CM})$. The UB tip is surrounded by the adjacent cap mesenchyme (CM, white and light grey). Wnt $9 b$ is expressed in the UB. The CM consists of an uninduced and an induced CM highlighted by gene expression patterns. Six2 is expressed in the whole CM, whereas only the uninduced CM (white) expresses Cited1. The induced CM (light grey) initiates Wnt 4 expression due to UB-derived Wnt9b. The induced $\mathrm{CM}$ transforms into a pre-tubular aggregate (dark grey) that separates from the CM. In the pre-tubular aggregate, Six 2 transcription is inhibited, whereas Wnt4 expression is induced by auto regulation

development. We review the literature covering the role of Wnt signaling in kidney development below.

\section{Induction of nephrogenesis by Wnt9b-mediated signaling}

Contact between the UB and CM is required for successful kidney development. Reciprocal signaling between these tissues triggers the differentiation of the $\mathrm{CM}$ into a nephron. Separation of the UB from the MM results in regression of both tissues in culture $[1,9]$. However, the isolated MM can be rescued from degeneration and induced to undergo nephrogenesis with exogenous signal sources.

When placed in contact with MM in culture, several embryonic tissues have the potential to provoke nephron differentiation in CM. The embryonic spinal cord (eSC) is the most potent one [10]. The capacity of the eSC to induce nephrogenesis is likely due to expression of Wnts [11], including Wnt4, which is a major endogenous Wnt present in induced CM [12]. Consistent with this, cell lines engineered to express other Wnts such as Wnt1, Wnt3a, Wnt4, Wnt7a, and Wnt7b were reported to trigger nephrogenesis in the isolated $\mathrm{MM}$ in a classical transfilter assay in which the MM and the Wnt-expressing cells are placed on the opposite sides of a porous polycarbonate filter [9].

Considerable effort was made to identify the UB-derived signal after the identification of Wnt4 as the major MMderived nephrogenesis control signal [12]. Both inorganic and biological factors were found that were able to elicit nephrogenesis in a cultured MM, such as lithium ions [13] 
and leukemia inhibitory factor (LIF) secreted by the UB [14]. Although LIF was found to be UB derived, its deficiency did not lead to fatal phenotype [15], which would be expected if primary nephrogenic induction were impaired. Wnt9b was eventually identified as a primary UB-derived factor that is able to induce the $\mathrm{CM}$ to exit from the stem cell stage and become permanently committed to the nephron cell lineages [16].

The $W n t 9 b$ gene is expressed in the epithelial Wolffian duct prior to induction of metanephros development. Wnt $9 \mathrm{~b}$ expression continues in the UB where it is more pronounced in the stalk region than in the tips (Figs. 1 and 3). The expression of $W n t 9 b$ in the mice is maintained in the collecting ducts until adulthood [17]. Wnt9b-mediated induction in the CM initiates expression of Wnt4, fibroblast growth factor $8(\mathrm{Fg} f 8)$, paired box 8 (Pax8), and LIM homeobox protein 1 (Lhx1)-encoding genes $[12,16,18-20]$. These genes fail to become expressed in the $\mathrm{CM}$ of $W n t 9 b$-deficient embryonic kidneys and no nephrons form [16], as a result, $W n t 9 b$ knockout mice die soon after birth. It becomes apparent that Wnt9b-mediated signaling from the $\mathrm{UB}$ to the $\mathrm{CM}$ is essential for the primary induction of the CM.

\section{Wnt4 provides a key nephrogenesis control signal downstream from Wnt9b}

The discovery that Wnt 4 function is necessary for formation of the pre-tubular aggregate and subsequent MET provided the first clue to the importance of Wnt signaling in kidney organogenesis [12]. Wnt9b-dependent Wnt4 expression appears in the induced CM and persists until the S-shaped body stage [7, 12, 16] (Fig. 3). Fate-mapping studies indicate that mature nephrons form entirely from the progeny of the cells that have expressed the Wnt4 gene [21]. Wnt4 function seems to be conserved because in the Xenopus laevis $x W n t 4$ is expressed in the pronephric anlage while later the expression becomes restricted to the tips of the pronephric tubule [22]. Similarly to the nephrons of mice, the pronephric tubules of Xenopus require $x W n t 4$ for their development [23].

Like $W n t 9 b$ deficiency, failure in Wnt4 signaling prevents nephrogenesis, and as a result only a vestigial kidney forms [12]. A mutation in the human Wnt4 gene associates with renal degeneration indicating a clinical relevance of this signal $[24$, 25]. The Wnt 4 knockout is characterized by a failure in nephron differentiation, which halts prior to formation of the pretubular aggregates. This histological anomaly can be associated with a stage during which the endogenous Wnt4 transcription is elevated. A non-functional Wnt4 mRNA remains transcribed in other tissues in the Wnt 4 knockout model, but the expression is lost from the CM. This suggests an autoinductive function of Wnt4 in the CM [12].

In the case of Wnt 4 deficiency, Pax8 and Fgf8 are initially expressed in few rudimentary pre-tubular aggregates [12, 20], which indicates that these factors function upstream of Wnt4 and downstream of Wnt9b (see above). Moreover, Wnt4 is not detected in the metanephros of the Fgf8-null mice, whereas Wnt4 expression remains in the Lhxl-deficient embryonic kidney [18-20] and Lhxl is absent in the kidney of both the Fgf8- and Wnt4-mutants. Collectively, the findings fit into a model in which, after the Wnt9b signaling, either Fgf8 and Wnt4 together or Wnt4 alone go on to induce the $L h x l$ gene in the pre-tubular aggregates. In any case, the Wnt4-dependent transcriptional program leading to $L h x l$ activation serves to differentiate the pre-tubular aggregate into the epithelialized renal vesicle. In short, Wnt4 acts in CM downstream of UBderived Wnt9b and is necessary for the formation of the pretubular aggregate and subsequent epithelial derivatives.

\section{Wnt signal transduction during nephron induction}

The downstream mechanisms elicited by Wnt signals in CM induction have been addressed in several studies. TCFreporter mice lines BAT-gal, TCF/Lef-lacZ, BATlacZ, and $T C F-g f p$ have demonstrated activation of the Wnt/TCF pathway in embryonic kidney [26-30]. These reporter lines revealed activity in the UB, but not in the MM and its derivatives [26, 29, 31-33]. However, another reporter line named TCF/Lef-lacZ [27] demonstrated TCF-mediated reporter activation in the distal S-shaped bodies and in the parietal epithelial layer of the Bowman's capsule [34, 35], so some discrepancies exist between reporter line studies.

In contrast to results from the reporter lines, the $\mathrm{Wnt} / \beta$ catenin/TCF pathway feedback target $L e f 1$ depicts the induced $\mathrm{CM}$ in a Wnt9b-dependent manner [7]. It is also noteworthy that the $\beta$-catenin-stabilizing small molecules (lithium chloride, $\mathrm{LiCl}$, and 5'-bromoindirubin-3'-oxime, $\mathrm{BIO}$ ), which both function by inhibiting GSK3 $\beta$, induce nephrogenesis in the separated MM and also provoke BATlacZ activity $[13,33,36$, 37]. $\mathrm{LiCl}$ induces expression of the $\mathrm{Wnt} / \beta$-catenin/TCF feedback genes Lef1 and Tcf1 and also Wnt4 in the isolated MM [37]. It is reasonable to conclude that activation of the $\beta$ catenin/TCF signaling pathway is sufficient to act upstream or at the level of Wnt4 to elicit a nephrogenesis response.

Knockout of $\beta$-catenin function with Six $2 \mathrm{Cre}$-mediated recombination was reported to perturb the nephron differentiation [38]. Analysis of such deficient embryonic kidneys demonstrated markedly reduced expression of $F g f 8, P a x 8$, Wht4, and Lhxl, pointing to a failure in CM induction [38]. In contrast, $\beta$-catenin gain-of-function in the $\mathrm{CM}$ with a constitutively active $\beta$-catenin leads to ectopic $\mathrm{CM}$ induction and premature depletion of the nephron stem cell pool. These responses occur independently of Wnt9b and Wnt4 function [38]. Interestingly, the sustained activation of $\beta$-catenin blocks MET, suggesting that $\beta$-catenin-mediated signaling has to be inhibited to complete the nephron development. Activating $\beta$-catenin mutations have been reported in Wilms 
tumors, which typically show an incompletely epithelialized blastemal component indicating a failure during early nephron differentiation [39, 40].

Recent studies have revealed that stabilization of $\beta$-catenin with BIO up-regulates $W n t 4$ and $F g f 8$ and suppresses Six2 expression in the aggregated MM cells [8]. Chromatin immunoprecipitation and sequencing indicated that the renal $\beta$ catenin protein binds to the cis-regulatory modules of the Wnt4, Fgf8, and Six 2 genes in the CM. These cis-regulatory elements also have TCF binding sites. In reporter studies of transgenic mice, the $W n t 4$ and $F g f 8$ cis-regulatory sequences driving $\beta$-galactosidase expression are able to promote the reporter expression when TCF sites are intact, whereas mutation of TCF motif impairs the $\beta$-galactosidase expression. This observation may be explained by $\beta$-catenin/TCF-mediated transcriptional up-regulation of the $W n t 4$ and $F g f 8$ genes in the CM derivatives. The observed reporter pattern by the Six 2 cis-regulatory module recapitulates the endogenous Six2 expression even if the TCF motif is mutated. This indicates that the suppression of the Six 2 associated with $\beta$-catenin accumulation is independent of the TCF motif [8]. In summary, $\beta$-catenin is sufficient to advance the nephron stem cell from their cell cycle to promote differentiation of the daughter cells towards the nephrons via up-regulation of $F g f 8$ and Wnt4. This induction stage is apparently controlled by Wnt9b via the $\beta$-catenin/TCF/Lef pathway and TCF/ Lef-independent $\beta$-catenin-mediated transcriptional program regulation.

\section{Downstream transduction of Wnt4 signaling}

After induction of the CM by Wnt9b, Wnt4 functions to promote MET [12]. Because the epithelization is inhibited by aberrant $\beta$-catenin signaling [38] and various TCF reporter lines report negative activity in $\mathrm{CM}$, alternative modes of Wnt4 signal transduction have been under scrutiny. Indeed, the Wnt/calcium/NFAT (nuclear factor of activated T cells) pathway may function as a downstream effector for Wnt4 signaling in the pre-tubular cell aggregates [32, 33]. Cytoplasmic calcineurin-dependent NFAT factors are expressed in early nephron forming cells, while the inhibitor for calcineurin inhibits nephrogenesis. Moreover, experimentally increased intracellular calcium in part rescues the Wnt4deficency phenotype by correcting failure in nephrogenesis [32]. Wnt4 can also induce a calcium influx and calcium/ calmodulin-dependent protein kinase II phosphorylation in primary MM cells [33]. This evidence suggests that Wnt4 induced by $W n t 9 b / \beta$-catenin functions in the $\mathrm{CM}$ at least in part via the calcium/NFAT pathway to promote MET. Lithium and BIO function probably upstream of Wnt 4 via $\beta$-catenin stabilization, whereas the calcium ionophores, which induce intracellular calcium concentration, may mimic the action of Wnt4 to promote nephrogenesis.

\section{Role of the planar cell polarity pathway in control of nephron maturation}

After the MET, the presumptive nephron becomes segmented and aligned into a unique corticomedullary oriented structure. Such complex architecture with defined tube length and diameter requires oriented cell division and coordinated cell motility. The Wnt signals have been shown to control these processes and also when deregulated to lead to pathophysiological changes and cystic kidney disease [41].

After the induction of the $\mathrm{CM}, \mathrm{Wnt} 9 \mathrm{~b}$ also has a role during later nephrogenesis in the coordination of the planar cell polarity and in cell intercalation during tubulogenesis via a process of convergent extension [17, 42]. Still, after birth, Wnt9b signaling has a role for oriented epithelial cell divisions to regulate the diameter of the epithelial tubules [17]. Renal cysts in Wnt9b-hypomorph mice are derived from the proximal convoluted tubules and attributed to disturbed activation of the Rho family GTPase (Rho) and Jun kinase, which are components of the Wnt/planar cell polarity (PCP) pathway [17].

Knockdown of Wnt/PCP components in frogs, such as dishevelled associated activator of morphogenesis 1 (Daam1), Rho guanine nucleotide exchange factor (WGEF), and $R h o$, consistently impairs the late morphogenesis of the pronephric tubules and duct. The pronephic cells of such morphants retain their ability for differentiation. This indicates that the Wnt/PCP pathway plays a role after the MET [43]. Studies of the tubular elongation in the Xenopus nephron with the time-lapse approach indicated convergent extension-type cellular movement during nephron maturation [42]. The process involves an evolutionarily conserved rosette-based cell organization. This is dependent on Wnt9b in mice and dishevelled-2 in the Xenopus [42]. It is reasonable to conclude that the Wnt/PCP signaling regulates nephron maturation after the initial induction and the stages of MET.

\section{The Wnt/ $\beta$-catenin pathway in nephron maturation}

Given that the Wnt/PCP pathway becomes important in nephron maturation and that the $\mathrm{Wnt} / \beta$-catenin signal transduction pathway needs to be suppressed to accomplish MET, mechanisms balancing the downstream signaling had to be explored. In addition to failure in MET and Wilms tumor association, incorrect activation of $\beta$-catenin signaling has been shown to cause renal cyst formation [44, 45], raising the possibility that cystic renal disease models may elucidate the factors behind downstream control of Wnt signaling in nephron maturation. A mouse line modeling nephronophthisis [46], a ciliopathy 
presenting with cystic renal disease, has provided a primary cilium protein named inversin as a potential Wnt signal transduction regulator candidate [47]. Inversin was shown to reduce the $\beta$-catenin amount by increasing proteasomal degradation of cytoplasmic dishevelled. Furthermore, inversin was demonstrated to promote $\beta$-catenin-independent Wnt signal transduction [47]. Finally, it has been speculated that the urine flow in early tubules may regulate the cellular response to Wnt signals via the primary cilium. Indeed, exposure of flow primary cells derived from the inner medullary collecting ducts increases inversin but decreases the level of $\beta$-catenin [47]. Another mechanism coordinating Wnt/b-catenin signaling inhibition functions after the renal vesicle stages and involves protein kinase A [48].

In addition to mutual organization, well-controlled differentiation of the nephron epithelium into highly specialized cell types is critical for kidney function. The specification of the proximodistal segmentation begins relatively early in morphogenesis as depicted by polarized expression of certain genes in the assembled renal vesicle [4]. The presumptive nephron contains progenitors for the parietal and visceral layers of the Bowman's capsule already during the S-shaped body stage [34]. $\beta$-catenin appears to be coupled to this control step as well, since conditional loss of its function in the nephron progenitors after the pre-tubular aggregate stage switches the fate of the parietal epithelial cells into the visceral phenotype presenting capillary connections [34]. Collectively, it appears that the Wnt signaling pathway is important during later nephron maturation stages after the MET. However, the downstream signal transduction involves suppression of the $\beta$ catenin branch and acquired dominance of the Wnt/PCP pathway. The switch between these pathways may be carried out by changes in the responsiveness of the tubular cells to endogenous Wnt ligands.

\section{Wnt11/GDNF signaling loop in control of the collecting duct system development}

The iterative UB bifurcation is a key determinant of the final nephron number and therefore of the size of the adult kidney. Glial cell line-derived neurotropic factor and Ret protooncogene (GDNF/Ret)-dependent signaling promotes formation of the UB from the Wolffian duct and subsequent branching during generation of the kidney collecting duct system. From the initial stages of kidney development, the CM expresses GDNF that signals to the UB via Ret to promote UB ingrowth and branch formations (Fig. 1). Deficiencies in either of these factors compromise kidney development due to growth failure of the UB [49-51].

Wnt11 functions with the GDNF/Ret to promote collecting duct development [52]. The Wnt11 gene is expressed at the UB tips [53, 54] (Fig. 1). Unlike Wnt1, Wnt3a, Wnt4, Wnt7a, and Wnt7b, Wnt11 fails to induce nephrogenesis in the MM explants [9]. Amphibian Wnt11b, which mammals have lost, can induce nephrogenic in the Xenopus pronephros [55]. As a result, it has been suggested that the loss of Wnt11b during evolution may be behind the regression of the mammalian pronephros [55]. Wnt11 deficiency in the mouse reduces kidney size and diminishes the number of nephrons [52]. The lack of Wnt1l function delays the first trifurcation event of the $\mathrm{UB}$ and was associated with a transient reduction in GDNF expression in the CM. In turn, exogenous GDNF promotes Wnt11 expression in the UB tips of isolated metanephric explants, supporting the finding that GDNF and Wnt11 are interdependent [56]. This notion is further supported by the findings that the few Ret homozygous mutant embryos that form the T-stage UB fail to express Wnt11 in the UB tips [52]. Furthermore, on a Ret heterozygous background the Wnt11 knockout allele reduces the number of nephrons dosedependently [52]. In mice, it appears Wnt11 and the GDNF/Ret pathways form a reciprocally positively regulated signal loop. This contributes to the generation of a sufficient number of UB branches to form the collecting duct system and to induce a corresponding amount of nephrons.

\section{The frizzled receptors in the kidney organogenesis}

Even though the essential role of the Wnt ligands in kidney organogenesis is well demonstrated, we have very limited knowledge of their receptors. Of the frizzled receptors at least $F z 2, F z 4, F z 6, F z 7, F z 8$, and $F z 10$ are expressed in mouse embryonic kidney [57, 58]. In Xenopus, morpholino-mediated knock down of $X f z 8$ impaired the epithelial morphogenesis of the pronephric duct and tubules [59].

In the mouse, compound $F z 4$ and $F z 8$ knock out allows the nephron to segment but the kidney size is reduced due to diminished proliferation and UB branching which resembles the phenotype of the Wnt11 knockout mice [52, 58]. The $F z 8$ knockout reduces the kidney size only when $F z 4$ function is impaired, indicating some redundancy between the frizzleds. Wnt11 may signal via fz 4 since this combination activates the TFC/Lef and the Rho reporters in cell culture. Concurrent expression of Wnt11 and Fz8 activates Rho suggesting specific mode of signaling via the frizzled subclasses [58]. Thus, Fz4 and Fz8 may act in synergy to transduce Wnt11 signaling and promote UB branching.

\section{Kidney medulla development involves Wnt7b and Wnt4 function}

In regard to kidney function, plasma filtration occurs in the cortex, while the osmoregulation of the filtrate occurs in the medulla where the Henle's loops and collecting ducts are 
located. The radially defined regions provide the foundations for the kidney to perform its secretory and excretory functions.

Of the $W n t$ genes, $W n t 7 b$ expressed in the stalk of the UB $[16,53]$, and Wnt7b-expressing cells can induce nephrogenesis in the separated MM tubule induction assay [9] (Fig. 1). However, knock out of $W n t 7 b$ in the UB cells leaves the nephrogenesis zone unaffected [60], whereas the medulla degenerates. In the Wnt4-, Fgf8-, and Lim 1-deficiency perturbing nephrogenesis, a well-distinguishable medullary still develops [12, 18-20]. It appears medulla formation is independent of the nephron formation process. This conclusion is further supported by the finding that the size of the Wnt $7 b$-deficient kidney remains close to normal and the number of UB tips remains unaffected. Consistent with the localization of $W n t 7 b$ mRNA, segmentation of the cortical nephrons occurs normally and the proximal and distal convoluted tubules and podocytes develop. However, the loops of Henle in the medulla are truncated without Wnt7b signaling. The proximal UB is also dilated, which can be attributed to changes in oriented cell divisions in the UB. Consistently, a deficiency in Wnt antagonist dickkopf-1 in the UB and developing nephrons leads to overgrowth of the renal medulla, suggesting that medullary development depends on a balance between Wnt7b and its antagonist dickkopf-1 [61].

In addition to the CM, the Wnt4 gene is expressed in the medullary stroma during kidney development. Here Wnt4 expression is not auto-regulated [53, 62]. Wnt4 knock out, however, reduces stromal Bmp4 expression and leads to absence of $\alpha$-smooth muscle actin-expressing cells, suggesting a failure in pericyte differentiation. This evidence suggests $\mathrm{Wn} 7 \mathrm{~b}$ and Wnt 4 are utilized as coordinating signals in developing kidney medulla.

\section{Downstream Wnt signaling factors in medulla development}

Nuclear translocation of $\beta$-catenin in embryonic kidney has been initially reported in human tissue [63]. Nuclear $\beta$-catenin is intense in the medullary stromal cells surrounding the collecting ducts of fetal human kidney. It turned out that the medullary-located periductal cells also express the Axin 2 and Lef1 Wnt signaling feedback targets [60]. Their expression is Wnt $7 \mathrm{~b}$ dependent, suggesting a response to the Wnt signaling [60]. Moreover, BAT-gal reporter demonstrated colocalization with Lef1 expression [60]. These observations can be considered highly suggestive for $\mathrm{Wnt} / \beta$-catenin/TCF pathway operation in embryonic kidney medullary cells. Consistent with this possibility, the inactivation of $\beta$-catenin function from the stromal cells with the aid of FoxD1Cre-mediated recombination generates a similar phenotype as that of $W n t 7 b$ knockout [60]. From this, we can conclude that the reciprocal interaction between the collecting ducts and the kidney stroma involves
Wnt $/ \beta$-catenin/TCF signaling, and this in turn coordinates the renal medulla development.

\section{Outlook for the future}

Wnt signaling is critical for several aspects of kidney organogenesis and it highlights the context-dependency of the downstream signaling systems. The key role of Wnt signaling in nephron induction and differentiation is well established, but the later morphogenic events that involve the PCP pathway need to be revealed in more detail. Given the severe phenotypes generated by the Wnt signaling system knock outs, failures in Wnt signal transduction will likely be associated with human congenital anomalies of the kidney and urinary tract (CAKUT). We expect to find more direct links between the renal Wnt signaling pathway and genetic diseases, which may lead to the identification of new signal transduction components and mechanisms. Of particular interest are the primary cilium and its role in regulating the responses to Wnt ligands in the tubular cells. Due to oftensubtle phenotype differences of the mutations that affect later stages of kidney organogenesis, novel methods such as threedimensional imaging and time-lapse techniques will aid the analysis.

There are Wnt ligands, such as Wnt2b, Wnt5a, and Wnt6, that are expressed in the embryonic kidney but of which the role remains open $[64,65]$. Wnt2b does not appear to be essential for tubule induction, since Wnt2/Wnt2b double knockout mice do not develop overt kidney phenotypes [66]. However, Wnt2b may function as a stromal signal to fine-tune UB morphogenesis [64, 67]. Wnt6 expression is detected in the UB and it has the capacity to induce nephron differentiation in the isolated MM [65], but no kidney-related phenotypes have been reported in the Wnt6-deficient mice.

Open Access This article is distributed under the terms of the Creative Commons Attribution License which permits any use, distribution, and reproduction in any medium, provided the original author(s) and the source are credited.

\section{References}

1. Saxen L (1987) Organogenesis of the kidney. Cambridge University Press

2. Mugford JW, Sipila P, McMahon JA, McMahon AP (2008) Osr1 expression demarcates a multi-potent population of intermediate mesoderm that undergoes progressive restriction to an Osr1dependent nephron progenitor compartment within the mammalian kidney. Dev Biol 324:88-98

3. Bard JB, Gordon A, Sharp L, Sellers WI (2001) Early nephron formation in the developing mouse kidney. J Anat 199:385-392 
4. Georgas K, Rumballe B, Valerius MT, Chiu HS, Thiagarajan RD, Lesieur E, Aronow BJ, Brunskill EW, Combes AN, Tang D, Taylor D, Grimmond SM, Potter SS, McMahon AP, Little MH (2009) Analysis of early nephron patterning reveals a role for distal RV proliferation in fusion to the ureteric tip via a cap mesenchymederived connecting segment. Dev Biol 332:273-286

5. Self M, Lagutin OV, Bowling B, Hendrix J, Cai Y, Dressler GR, Oliver G (2006) Six2 is required for suppression of nephrogenesis and progenitor renewal in the developing kidney. EMBO J 25:52145228

6. Kobayashi A, Valerius MT, Mugford JW, Carroll TJ, Self M, Oliver G, McMahon AP (2008) Six2 defines and regulates a multipotent self-renewing nephron progenitor population throughout mammalian kidney development. Cell Stem Cell 3:169-181

7. Mugford JW, Yu J, Kobayashi A, McMahon AP (2009) Highresolution gene expression analysis of the developing mouse kidney defines novel cellular compartments within the nephron progenitor population. Dev Biol 333:312-323

8. Park JS, Ma W, O'Brien LL, Chung E, Guo JJ, Cheng JG, Valerius MT, McMahon JA, Wong WH, McMahon AP (2012) Six2 and Wnt regulate self-renewal and commitment of nephron progenitors through shared gene regulatory networks. Dev Cell 23:637-651

9. Kispert A, Vainio S, McMahon AP (1998) Wnt-4 is a mesenchymal signal for epithelial transformation of metanephric mesenchyme in the developing kidney. Development 125:4225-4234

10. Grobstein C (1956) Trans-filter induction of tubules in mouse metanephrogenic mesenchyme. Exp Cell Res 10:424-440

11. Parr BA, Shea MJ, Vassileva G, McMahon AP (1993) Mouse Wnt genes exhibit discrete domains of expression in the early embryonic CNS and limb buds. Development 119:247-261

12. Stark K, Vainio S, Vassileva G, McMahon AP (1994) Epithelial transformation of metanephric mesenchyme in the developing kidney regulated by wnt- 4 . Nature 372:679-683

13. Davies JA, Garrod DR (1995) Induction of early stages of kidney tubule differentiation by lithium ions. Dev Biol 167:50-60

14. Barasch J, Yang J, Ware CB, Taga T, Yoshida K, Erdjument-Bromage H, Tempst P, Parravicini E, Malach S, Aranoff T, Oliver JA (1999) Mesenchymal to epithelial conversion in rat metanephros is induced by LIF. Cell 99:377-386

15. Stewart CL, Kaspar P, Brunet LJ, Bhatt H, Gadi I, Kontgen F, Abbondanzo SJ (1992) Blastocyst implantation depends on maternal expression of leukaemia inhibitory factor. Nature 359:76-79

16. Carroll TJ, Park JS, Hayashi S, Majumdar A, McMahon AP (2005) Wnt9b plays a central role in the regulation of mesenchymal to epithelial transitions underlying organogenesis of the mammalian urogenital system. Dev Cell 9:283-292

17. Karner CM, Chirumamilla R, Aoki S, Igarashi P, Wallingford JB, Carroll TJ (2009) Wnt9b signaling regulates planar cell polarity and kidney tubule morphogenesis. Nat Genet 41:793-799

18. Grieshammer U, Cebrian C, Ilagan R, Meyers E, Herzlinger D, Martin GR (2005) FGF8 is required for cell survival at distinct stages of nephrogenesis and for regulation of gene expression in nascent nephrons. Development 132:3847-3857

19. Kobayashi A, Kwan KM, Carroll TJ, McMahon AP, Mendelsohn CL, Behringer RR (2005) Distinct and sequential tissue-specific activities of the LIM-class homeobox gene Lim1 for tubular morphogenesis during kidney development. Development 132:2809-2823

20. Perantoni AO, Timofeeva O, Naillat F, Richman C, PajniUnderwood S, Wilson C, Vainio S, Dove LF, Lewandoski M (2005) Inactivation of FGF8 in early mesoderm reveals an essential role in kidney development. Development 132:3859-3871

21. Shan J, Jokela T, Skovorodkin I, Vainio S (2010) Mapping of the fate of cell lineages generated from cells that express the Wnt 4 gene by time-lapse during kidney development. Differentiation 79:57-64
22. Carroll TJ, Wallingford JB, Vize PD (1999) Dynamic patterns of gene expression in the developing pronephros of Xenopus laevis. Dev Genet 24:199-207

23. Saulnier DM, Ghanbari H, Brandli AW (2002) Essential function of wnt-4 for tubulogenesis in the Xenopus pronephric kidney. Dev Biol 248:13-28

24. Biason-Lauber A, Konrad D, Navratil F, Schoenle EJ (2004) A WNT4 mutation associated with Müllerian-duct regression and virilization in a 46, XX woman. N Engl J Med 351:792-798

25. Mandel H, Shemer R, Borochowitz ZU, Okopnik M, Knopf C, Indelman M, Drugan A, Tiosano D, Gershoni-Baruch R, Choder M, Sprecher E (2008) SERKAL syndrome: an autosomal-recessive disorder caused by a loss-of-function mutation in WNT4. Am J Hum Genet 82:39-47

26. Maretto S, Cordenonsi M, Dupont S, Braghetta P, Broccoli V, Hassan AB, Volpin D, Bressan GM, Piccolo S (2003) Mapping Wnt/betacatenin signaling during mouse development and in colorectal tumors. Proc Natl Acad Sci USA 100:3299-3304

27. Mohamed OA, Clarke HJ, Dufort D (2004) Beta-catenin signaling marks the prospective site of primitive streak formation in the mouse embryo. Dev Dyn 231:416-424

28. Nakaya MA, Biris K, Tsukiyama T, Jaime S, Rawls JA, Yamaguchi TP (2005) Wnt3a links left-right determination with segmentation and anteroposterior axis elongation. Development 132:5425-5436

29. Ferrer-Vaquer A, Piliszek A, Tian G, Aho RJ, Dufort D, Hadjantonakis AK (2010) A sensitive and bright single-cell resolution live imaging reporter of Wnt/s-catenin signaling in the mouse. BMC Dev Biol 10:121

30. Barolo S (2006) Transgenic Wnt/TCF pathway reporters: all you need is lef? Oncogene 25:7505-7511

31. Schmidt-Ott KM, Masckauchan TN, Chen X, Hirsh BJ, Sarkar A, Yang J, Paragas N, Wallace VA, Dufort D, Pavlidis P, Jagla B, Kitajewski J, Barasch J (2007) Beta-catenin/TCF/lef controls a differentiation-associated transcriptional program in renal epithelial progenitors. Development 134:3177-3190

32. Burn SF, Webb A, Berry RL, Davies JA, Ferrer-Vaquer A, Hadjantonakis AK, Hastie ND, Hohenstein P (2011) Calcium/ NFAT signalling promotes early nephrogenesis. Dev Biol 352:288298

33. Tanigawa S, Wang H, Yang Y, Sharma N, Tarasova N, Ajima R, Yamaguchi TP, Rodriguez LG, Perantoni AO (2011) Wnt4 induces nephronic tubules in metanephric mesenchyme by a non-canonical mechanism. Dev Biol 352:58-69

34. Grouls S, Iglesias DM, Wentzensen N, Moeller MJ, Bouchard M, Kemler R, Goodyer P, Niggli F, Grone HJ, Kriz W, Koesters R (2012) Lineage specification of parietal epithelial cells requires -catenin/Wnt signaling. J Am Soc Nephrol 23:63-72

35. Iglesias DM, Hueber PA, Chu L, Campbell R, Patenaude AM, Dziarmaga AJ, Quinlan J, Mohamed O, Dufort D, Goodyer PR (2007) Canonical WNT signaling during kidney development. Am J Physiol Ren Physiol 293:F494-F500

36. Klein PS, Melton DA (1996) A molecular mechanism for the effect of lithium on development. Proc Natl Acad Sci USA 93:8455-8459

37. Kuure S, Popsueva A, Jakobson M, Sainio K, Sariola H (2007) Glycogen synthase kinase-3 inactivation and stabilization of betacatenin induce nephron differentiation in isolated mouse and rat kidney mesenchymes. J Am Soc Nephrol 18:1130-1139

38. Park JS, Valerius MT, McMahon AP (2007) Wnt/beta-catenin signaling regulates nephron induction during mouse kidney development. Development 134:2533-2539

39. Koesters R, Ridder R, Kopp-Schneider A, Betts D, Adams V, Niggli F, Briner J, von Knebel DM (1999) Mutational activation of the betacatenin proto-oncogene is a common event in the development of Wilms tumors. Cancer Res 59:3880-3882

40. Md Zin R, Murch A, Charles A (2011) Pathology, genetics and cytogenetics of Wilms tumour. Pathology 43:302-312 
41. Lancaster MA, Gleeson JG (2010) Cystic kidney disease: the role of Wnt signaling. Trends Mol Med 16:349-360

42. Lienkamp SS, Liu K, Karner CM, Carroll TJ, Ronneberger O, Wallingford JB, Walz G (2012) Vertebrate kidney tubules elongate using a planar cell polarity-dependent, rosette-based mechanism of convergent extension. Nat Genet 44:1382-1387

43. Miller RK, Canny SG, Jang CW, Cho K, Ji H, Wagner DS, Jones EA, Habas R, McCrea PD (2011) Pronephric tubulogenesis requires Daam1-mediated planar cell polarity signaling. J Am Soc Nephrol 22:1654-1664

44. Saadi-Kheddouci S, Berrebi D, Romagnolo B, Cluzeaud F, Peuchmaur M, Kahn A, Vandewalle A, Perret C (2001) Early development of polycystic kidney disease in transgenic mice expressing an activated mutant of the beta-catenin gene. Oncogene 20:5972-5981

45. Qian CN, Knol J, Igarashi P, Lin F, Zylstra U, Teh BT, Williams BO (2005) Cystic renal neoplasia following conditional inactivation of apc in mouse renal tubular epithelium. J Biol Chem 280:3938-3945

46. Hildebrandt F, Attanasio M, Otto E (2009) Nephronophthisis: disease mechanisms of a ciliopathy. J Am Soc Nephrol 20:23-35

47. Simons M, Gloy J, Ganner A, Bullerkotte A, Bashkurov M, Kronig C, Schermer B, Benzing T, Cabello OA, Jenny A, Mlodzik M, Polok B, Driever W, Obara T, Walz G (2005) Inversin, the gene product mutated in nephronophthisis type II, functions as a molecular switch between Wnt signaling pathways. Nat Genet 37:537-543

48. Gallegos TF, Kouznetsova V, Kudlicka K, Sweeney DE, Bush KT, Willert K, Farquhar MG, Nigam SK (2012) A protein kinase A and Wnt-dependent network regulating an intermediate stage in epithelial tubulogenesis during kidney development. Dev Biol 364:11-21

49. Schuchardt A, D'Agati V, Larsson-Blomberg L, Costantini F, Pachnis $\mathrm{V}$ (1994) Defects in the kidney and enteric nervous system of mice lacking the tyrosine kinase receptor ret. Nature 367:380-383

50. Schuchardt A, D'Agati V, Pachnis V, Costantini F (1996) Renal agenesis and hypodysplasia in ret-k- mutant mice result from defects in ureteric bud development. Development 122:1919-1929

51. Sanchez MP, Silos-Santiago I, Frisen J, He B, Lira SA, Barbacid M (1996) Renal agenesis and the absence of enteric neurons in mice lacking GDNF. Nature 382:70-73

52. Majumdar A, Vainio S, Kispert A, McMahon J, McMahon AP (2003) Wnt11 and ret/gdnf pathways cooperate in regulating ureteric branching during metanephric kidney development. Development 130:3175-3185

53. Kispert A, Vainio S, Shen L, Rowitch DH, McMahon AP (1996) Proteoglycans are required for maintenance of wnt-11 expression in the ureter tips. Development 122:3627-3637

54. Lako M, Strachan T, Bullen P, Wilson DI, Robson SC, Lindsay S (1998) Isolation, characterisation and embryonic expression of WNT11, a gene which maps to 11q13.5 and has possible roles in the development of skeleton, kidney and lung. Gene 219:101-110
55. Tetelin S, Jones EA (2010) Xenopus Wnt11b is identified as a potential pronephric inducer. Dev Dyn 239:148-159

56. Pepicelli CV, Kispert A, Rowitch DH, McMahon AP (1997) GDNF induces branching and increased cell proliferation in the ureter of the mouse. Dev Biol 192:193-198

57. McMahon AP, Aronow BJ, Davidson DR, Davies JA, Gaido KW, Grimmond S, Lessard JL, Little MH, Potter SS, Wilder EL, Zhang P (2008) GUDMAP: the genitourinary developmental molecular anatomy project. J Am Soc Nephrol 19:667-671

58. Ye X, Wang Y, Rattner A, Nathans J (2011) Genetic mosaic analysis reveals a major role for frizzled 4 and frizzled 8 in controlling ureteric growth in the developing kidney. Development 138:1161-1172

59. Satow R, Chan TC, Asashima M (2004) The role of Xenopus frizzled8 in pronephric development. Biochem Biophys Res Commun 321: 487-494

60. Yu J, Carroll TJ, Rajagopal J, Kobayashi A, Ren Q, McMahon AP (2009) A Wnt7b-dependent pathway regulates the orientation of epithelial cell division and establishes the cortico-medullary axis of the mammalian kidney. Development 136:161-171

61. Pietila I, Ellwanger K, Railo A, Jokela T, Barrantes Idel B, Shan J, Niehrs C, Vainio SJ (2011) Secreted Wnt antagonist dickkopf-1 controls kidney papilla development coordinated by wnt-7b signalling. Dev Biol 353:50-60

62. Itaranta P, Chi L, Seppanen T, Niku M, Tuukkanen J, Peltoketo H, Vainio S (2006) Wnt-4 signaling is involved in the control of smooth muscle cell fate via bmp-4 in the medullary stroma of the developing kidney. Dev Biol 293:473-483

63. Eberhart CG, Argani P (2001) Wnt signaling in human development: Beta-catenin nuclear translocation in fetal lung, kidney, placenta, capillaries, adrenal, and cartilage. Pediatr Dev Pathol 4:351-357

64. Lin Y, Liu A, Zhang S, Ruusunen T, Kreidberg JA, Peltoketo H, Drummond I, Vainio S (2001) Induction of ureter branching as a response to wnt-2b signaling during early kidney organogenesis. Dev Dyn 222:26-39

65. Itaranta P, Lin Y, Perasaari J, Roel G, Destree O, Vainio S (2002) Wnt-6 is expressed in the ureter bud and induces kidney tubule development in vitro. Genesis: J Genet Dev 32:259-268

66. Goss AM, Tian Y, Tsukiyama T, Cohen ED, Zhou D, Lu MM, Yamaguchi TP, Morrisey EE (2009) Wnt2/2b and beta-catenin signaling are necessary and sufficient to specify lung progenitors in the foregut. Dev Cell 17:290-298

67. Hatini V, Huh SO, Herzlinger D, Soares VC, Lai E (1996) Essential role of stromal mesenchyme in kidney morphogenesis revealed by targeted disruption of winged helix transcription factor BF-2. Genes Dev 10:1467-1478 